Factibilidad de creación de un supermercado en el cantón Muisne de la provincia de Esmeraldas

\title{
Factibilidad de creación de un supermercado en el cantón Muisne de la provincia de Esmeraldas
}

\section{Feasibility of creating a supermarket in the Muisne canton of the province of Esmeraldas}

\section{Viabilidade de criação de um supermercado no cantão de Muisne, na província de Esmeraldas}

\author{
Mirna C. Bedoya-Flores ${ }^{\mathrm{I}}$ \\ mirnabedoya@hotmail.com \\ Rugina Elidea Quiñonez II \\ rugina.quinonez@hotmail.com \\ Manuel A. Plaza-Castillo III \\ plazita66@hotmail.com
}

Recibido: 18 de enero de 2018 * Corregido: 23 de marzo de $2018 *$ Aceptado: 05 de junio de 2018

I. Ingeniera en Contabilidad y Auditoría CPA, Docente de la Universidad Técnica "Luis Vargas Torres" de Esmeraldas, Esmeraldas, Ecuador.

II. Magister en Administración de Empresas, Ingeniera en Administración Pública, Tecnólogo en Administración Pública, Docente de la Universidad Técnica "Luis Vargas Torres" de Esmeraldas, Esmeraldas, Ecuador.

min. Magister en Gestión Ambiental, Magister en Administración de Empresas, Licenciado en Administración de Empresas, Ingeniero Comercial, Docente de la Universidad Técnica "Luis Vargas Torres" de Esmeraldas, Esmeraldas, Ecuador. 
Factibilidad de creación de un supermercado en el cantón Muisne de la provincia de Esmeraldas

\section{Resumen}

El presente artículo pretende dar a conocer una posible solución a una problemática de escasa cobertura de productos de primera necesidad y complementarios que satisfagan a los diferentes clientes y consumidores localizados en la zona urbana y rural del cantón Muisne, Para ello se propone estudiar la factibilidad para la creación de un supermercado en dicha provincia. El tipo de investigación fue documental - descriptivo con un diseño no experimental. La recolección de la información se obtuvo a través de un instrumento tipo cuestionario y la técnica utilizada fue la encuesta. La población fue constituida por un total de 1480 familias con 4 o 5 miembros y el cálculo de la muestra fue de 472. Los datos obtenidos se procesaron considerando una estadística de tipo descriptiva. Los resultados evidenciaron que el 99,6\% de los investigados coincidieron en opinar acerca de la necesidad de implementar un supermercado que satisfaga sus necesidades. Entre sus conclusiones se determinó que ante el comportamiento de compras de las personas que habitan en Muisne y la alta aceptación por los habitantes, se requiere la estructuración de un supermercado que esté acorde a sus necesidades, siendo un mercado estratégico que se espera aprovechar y explotar.

Palabras clave: factibilidad; supermercado; demanda; oferta y productos alimenticios.

\section{Abstract}

This article seeks to highlight a possible solution to a problem of low coverage of staples and complementary satisfy different customers and consumers located in the urban and rural area of the canton Muisne, this is proposed to study the feasibility for the creation of a supermarket in this province. The type of investigation was documentary - descriptive with a non-experimental design. The collection of information was obtained through a questionnaire type instrument and the technique used was the survey. The population was constituted by a total of 1480 families with 4 or 5 members and calculation of the sample was 472 . The data obtained were processed considering a descriptive statistic. The results showed that $99.6 \%$ of the investigated agreed to review about the need for a supermarket that meets your needs. Among its conclusions was determined that the behavior of people shopping that live in Muisne and high acceptance by the inhabitants, is required the structuring of a supermarket that is commensurate with their needs, being a strategic market to be expected to take advantage of and exploit.

Keywords: feasibility; supermarket; demand; supply; and food products. 
Factibilidad de creación de un supermercado en el cantón Muisne de la provincia de Esmeraldas

\section{Resumo}

Este artigo tem como objetivo apresentar uma possível solução para um problema de escassa cobertura de necessidades complementares e básicas que satisfaçam os diferentes clientes e consumidores localizados na zona urbana e rural do cantão de Muisne, para isso propõe-se estudar a viabilidade de a criação de um supermercado na referida província. O tipo de pesquisa foi documentário - descritivo com delineamento não experimental. As informações foram coletadas por meio de um instrumento do tipo questionário e a técnica utilizada foi a pesquisa. A população foi constituída por um total de 1480 famílias com 4 ou 5 membros e o cálculo da amostra foi de 472 . Os dados obtidos foram processados considerando uma estatística descritiva do tipo. Os resultados mostraram que 99,6\% dos entrevistados concordaram com a necessidade de implementar um supermercado que atenda às suas necessidades. Entre suas conclusões, determinou-se que, tendo em vista o comportamento de compra das pessoas que vivem em Muisne e a alta aceitação dos habitantes, é necessária a estruturação de um supermercado que esteja de acordo com suas necessidades, sendo um mercado estratégico que deve ser explorado e explorado.

Palavras chave: viabilidade; supermercado demanda oferta e produtos alimentícios.

\section{Introducción}

Desde el punto de vista analítico, los supermercados a través del tiempo se han ido extendiendo en el territorio ecuatoriano, ofertando diversos productos con valor agregado para satisfacer las necesidades de los consumidores. La tecnología es un factor que permite a los supermercados evolucionar tanto en innovación como en la comercialización de las variedades de productos, con precios direccionados en base a los diferentes tipos de mercado que se quieran satisfacer con insumos de primera necesidad. En el cantón Muisne, desde sus inicios los negocios de tiendas de abarrotes han sido regularmente pequeños, pero algunos han ido creciendo paulatinamente en infraestructura y en variedad de mercaderías para comercializarla; estos son quienes a su vez conforman la oferta que satisfacen las necesidades de las personas que habitan en el sector.

Los administradores de los negocios en pleno crecimiento solo adquieren y ofrecen productos clásicos, limitando la oportunidad a encontrar variedad en los artículos de primera necesidad para así poder dar al consumidor una elección al momento de realizar sus compras. Además, algunos negocios por su 
Factibilidad de creación de un supermercado en el cantón Muisne de la provincia de Esmeraldas

tamaño determinan el nivel de precios que en la mayoría de los casos elevan el costo de vida de la población.

La mayor parte de las personas que tienen negocios dedicados a la venta de artículos de primera necesidad proviene de la Sierra; que, por buscar un nuevo mercado para vender productos en estado natural como verduras, frutas, hortalizas, entre otros; se dieron cuenta de la oportunidad que había, decidiendo radicarse en Muisne para dedicarse de manera regular a la venta de productos naturales y procesados para ofrecer a los hogares del cantón. Las estadísticas muestran que Muisne ha tenido bajo desarrollo en el ámbito económico, político, social, es por ello que se puede apreciar la poca transcendencia de las actividades productivas en general y específicamente en la comercial.

De acuerdo a los datos de la población del censo del año 2010, los grupos de familia en promedio son de seis miembros, existen cerca de 1480 hogares en Muisne, los establecimientos de expendio de alimentos actualmente no cubren todas las necesidades básicas de los habitantes de este cantón, por lo que estas familias deben enfrentarse al éxodo de ir a otros lugares para surtir su canasta básica o víveres aminorando su capacidad de compra, ya que necesitan de varios medios de transporte para movilizarse a otros sectores y obtener los productos de primera necesidad, entre esos sitios de afluencia está el cantón Atacames a 60 minutos de distancia y la ciudad de Esmeraldas a 90 minutos. Por todo lo expuesto, en este artículo se estudia la factibilidad para la creación de un supermercado en el cantón Muisne de la provincia de Esmeraldas que permita satisfacer la escasa cobertura de productos de primera necesidad y complementarios en los diferentes clientes y consumidores localizados en la zona urbana y rural del cantón Muisne, conformado por los diferentes tipos de estratos sociales, dado que en los comerciales de abarrotes, tiendas o despensas no se cumple o satisfacen las expectativas de los clientes.

Según la revista LÍDERES.EC el analista de investigación Oster (2013) analizando ¿Cuánto puede influir en el crecimiento de los supermercados el alza del Bono de Desarrollo Humano, de USD 35 a 50 ? El incremento del bono y su relación con el crecimiento de los supermercados puede ser variable; por un lado, las personas tendrán más ingresos y podrían aumentar el monto de compra en el supermercado. Sin embargo, es difícil indicar hasta qué punto beneficiaría a los supermercados, ya que el aumento no será destinado solo a compras de alimentos, sino también a otros gastos de los 
Factibilidad de creación de un supermercado en el cantón Muisne de la provincia de Esmeraldas

beneficiarios, como movilización, vestimenta, salud, entre otros. De todas maneras, el hecho de que las personas de bajos recursos tengan un mayor ingreso sí beneficiará las ventas de los supermercados. ¿A qué se refiere cuando menciona a los supermercados más pequeños'? Básicamente, están en zonas donde predominan las tiendas de barrio y en donde las personas no tienen acceso a un supermercado. Se ubican en sitios altamente poblados, y la idea es evitar que las personas se movilicen largas distancias, que tengan un fácil acceso a productos de calidad a un precio más bajo que las tiendas. Estos supermercados compiten directamente con las tiendas de barrio.

De allí que, los supermercados se han convertido en lugares estratégicos porque expenden productos de consumo masivo, satisfaciendo necesidades de los habitantes del sector donde se encuentren, anteriormente estos lugares eran visitados mayormente por personas con estabilidad económica media-alta, pero actualmente también son visitadas por las clases bajas, probablemente por los precios económicos que estos lugares ofertan a este segmento de personas; también se dieron cuenta que es un mercado estratégico al que pueden abarcar, ofreciendo productos que frecuentemente consumen estas clases de personas.

\section{Distribución de productos y secciones en un supermercado}

Sánchez (2013), en el estudio realizado sobre los supermercados indica que lo primero que se debe plantear es el tipo de producto, y su forma de almacenamiento, es decir si es lácteo o carnes, debe ir refrigerado, si es tóxico o tiene un olor específico debe estar en un lugar ventilado, si se daña con el sol o el calor es necesario colocarlo en un lugar fresco etc.; existen clasificaciones estandarizadas de la distribución de los productos de gran consumo, tanto de alimentación como de no alimentación, utilizable en todos los circuitos comerciales: hipermercados, supermercados, multiespecialistas, especialistas y mayoristas.

En cuanto a la ubicación de los productos las técnicas de marketing comienzan a actuar desde el mismo momento en el que se busca un lugar para la ubicación del establecimiento, hasta llegar a la venta de la mercadería que se comercializará; dentro del supermercado se encontrará que casi en toda la superficie se extenderá por la izquierda y en la parte derecha tan sólo habrá un espacio en el que habitualmente colocarán ropa, la razón no es otra, de que usted probablemente, no entrará al supermercado a comprar un vestido o un pantalón pero el efecto que se consigue es que cuando el comprador haya mirado a la derecha es posible que tenga visto algo que le interese. Los expertos son 
Factibilidad de creación de un supermercado en el cantón Muisne de la provincia de Esmeraldas

conscientes de que el cliente irá hacia el fondo y luego, inevitablemente, tendrá que dirigirse hacia la izquierda. Por eso, colocan los alimentos en esas direcciones.

Pero como lo que quieren los comerciantes es que el cliente se pasee toda la superficie, dispersan por todo el establecimiento los alimentos de primera necesidad. Las zonas en las que se divide el establecimiento son calientes y frías; en la zona caliente está asegurada la circulación de los clientes, así, los productos que se exponen son los de menor compra, mientras que en la zona fría hay que inducir a la compra, por lo que se colocan artículos de primera necesidad.

Es claro notar que según lo que indica Sánchez (2013) es valedero, ya que para administrar un negocio de este tipo hay que saberlo hacer; las cosas no se dan por simple coincidencia, sino que hasta para colocar un mínimo detalle dentro de los supermercados tiene su significado, en negocios como estos hay que buscar la manera de vender hasta lo más mínimo, claro está dependiendo de las necesidades de los consumidores.

\section{Mezcla de mercadotecnia (marketing mix)}

Según McCarthy (2005) el concepto de "las 4 P's como son producto, precio, plaza y promoción, que hoy por hoy, se constituye en la clasificación más utilizada para estructurar las herramientas o variables de la mezcla de mercadotecnia".

Producto: es el conjunto de atributos tangibles o intangibles que la empresa ofrece al mercado meta.

Precio: Se entiende como la cantidad de dinero que los clientes tienen que pagar por un determinado producto o servicio; el precio representa la única variable de la mezcla de mercadotecnia que genera ingresos para la empresa.

Plaza: También conocida como Posición o Distribución, incluye todas aquellas actividades de la empresa que ponen el producto a disposición del mercado meta.

Promoción: Abarca una serie de actividades cuyo objetivo es: informar, persuadir y recordar las características, ventajas y beneficios del producto.

Las diferentes promociones que se pueden practicar en la implementación del supermercado pueden ser:

Tarjetas de crédito.

Tarjetas de afiliación

Descuentos en roles 
Factibilidad de creación de un supermercado en el cantón Muisne de la provincia de Esmeraldas

La mezcla de mercadotecnia es un elemento que permite estructurar planes de marketing en las empresas tanto grandes, medianas y pequeñas, abriendo nuevas visiones de los diferentes escenarios para los negocios, las cuatro P's permiten que cada uno de los administradores y propietarios escojan las más adecuada para la utilización de su negocio; para la implementación de presente proyecto dichas estrategias se determinarán en el transcurso de la de la investigación.

\section{Satisfacción del cliente y sus beneficios}

Esto puede apegarse a lo que indica Philip (2005) en su investigación de la satisfacción del cliente como "el nivel del estado de ánimo de una persona que resulta de comparar el rendimiento percibido de un producto o servicio con sus expectativas. Algunos de los beneficios de esta satisfacción son: El cliente satisfecho, por lo general, vuelve a comprar. Por tanto, la empresa obtiene como beneficio su lealtad y por ende la posibilidad de venderle el mismo u otros productos adicionales en el futuro. El cliente satisfecho comunica a otros sus experiencias positivas con un producto o servicio. Por tanto, la empresa obtiene como beneficio una difusión gratuita que el cliente satisfecho realiza a sus familiares, amistades y conocidos.

El cliente satisfecho deja de lado a la competencia. Por tanto, la empresa obtiene como beneficio un determinado lugar (participación) en el mercado.

\section{Estudio de mercado}

Según Urbina (2010), “es la determinación y cuantificación de la demanda y la oferta, el análisis de los precios y el estudio de la comercialización, y se determina la primera parte de investigación formal del estudio; también el estudio de Mercado es útil para prever una política adecuada de precios" (p.7) Es necesario en Mercadeo hacer un estudio de Mercado que pueda garantizar que las estrategias que se decidan en los negocios corresponden a lo que el negocio necesita para mejorar su posición en el mercado.

\section{Estudio técnico}

Según Urbina (2010), "En este estudio se presenta la determinación del tamaño óptimo de la planta de la localización, la ingeniería del proyecto y el análisis organizativo, administrativo y legal”. (p.7) El estudio técnico es la investigación que permite identificar el requerimiento sobre: ¿Cuánto se necesita para la constitución de la empresa?, ¿Qué se dará o se está pensando hacer?, ¿Cómo se llevará a cabo la infraestructura?, con las implementaciones necesarias. Son los elementos técnicos de 
Factibilidad de creación de un supermercado en el cantón Muisne de la provincia de Esmeraldas

instalación, que garantizaran la distribución adecuada de los productos según las necesidades de los clientes a atender.

\section{Oferta}

Urbina (2010) indica que "es la cantidad de bienes o servicios que un cierto número de oferentes (productores) está dispuesto a poner a disposición del mercado a un precio determinado.” (p.41). La oferta mide las cantidades y estado en que la economía de las pequeñas y medianas empresas puede poner a disposición del mercado bienes o servicios, esta oferta es determinada muchas veces por la cantidad de personas que habitan la zona o que concurren a la misma, un gran apoyo para esta decisión lo constituye el estudio de mercado.

\section{Estrategias de mercado}

Esto puede apegarse, con lo que indica Santillán (2012), en su tesis de grado (p.18) Tarjeta de Afiliación: Dirigida a sus clientes para verificar sus saldos disponibles en el momento que se precise o al realizar sus compras, mantener línea directa con todas las tarjetas de crédito, optimizando el tiempo de espera en las cajas, y facilitando la compra de los alimentos. Dentro de los servicios se incluye también órdenes de compra y bonos navideños, que son documentos valorados que se emiten a personas, empresas, por la denominación deseada, que permite a los usuarios realizar compras a precio de afiliado.

Las estrategias de Mercadeo son necesarias al momento de administrar un negocio ya que son instrumentos necesarios y principales que se deben utilizar para la puesta en marcha de una empresa o negocio, si no se utilizan buenas estrategias no se obtendrá la rentabilidad o el perfeccionamiento que puede ir obteniendo cada día en la atención de la empresa, y principalmente la lealtad del usuario.

\section{Metodología}

El método que se aplicó principalmente en este estudio fue de tipo deductivo - inductivo. Con el primero se analizó el estado en general por el que pasa esta localidad al no contar con un supermercado; y a través de eso, se pudo establecer los efectos e impactos que den en este lugar; luego, a partir del método inductivo se pudo comprobar de manera puntual el comportamiento de las tiendas de abarrotes de Muisne, en cuanto a lo relacionado con la variedad de productos, el precio y atención al cliente. 
Factibilidad de creación de un supermercado en el cantón Muisne de la provincia de Esmeraldas

El tipo de investigación fue documental - descriptivo y con un diseño no experimental. La recolección de la información se obtuvo a través de un instrumento tipo cuestionario y la técnica utilizada fue la encuesta, posterior a la observación de forma visual al momento de realizar las encuestas a los habitantes de Muisne y entrevistas a los distintos locales comerciales. Así mismo se realizó una entrevista tipo encuestas con un banco de preguntas para cada una de las autoridades que regulan el funcionamiento administrativo y legal de las instituciones y negocios de Muisne. La población fue constituida por un total de 1480 familias con 4 o 5 miembros y el cálculo de la muestra fue de 472 . Los datos obtenidos se procesaron considerando una estadística de tipo descriptiva.

\section{Análisis e interpretación de los resultados}

Al segmentar el mercado, de acuerdo a los datos aportados por las familias investigadas se pudo constatar que no se encuentra apropiadamente atendido y que al implementar un supermercado en esa localidad tendrá un 99,6\% de aceptación, además, realizarán sus compras en el supermercado.

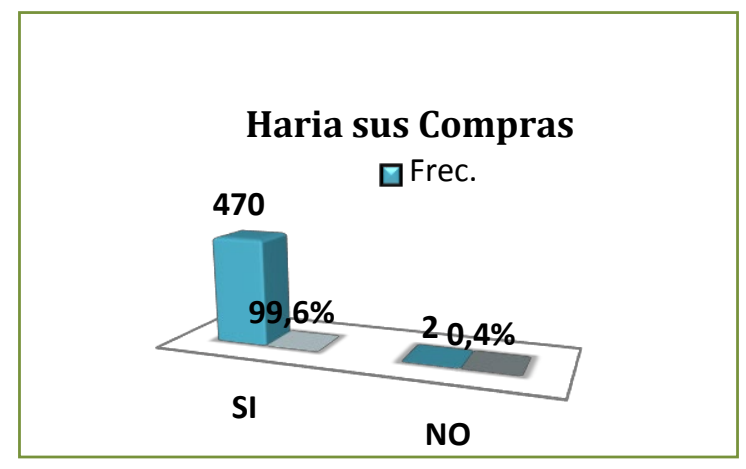

Grafico 1. Segmentación del mercado

Fuente: elaboración propia.

En relación a la oferta, en el cantón Muisne se han ido incrementando negocios de expendio de productos alimenticios como tiendas de barrio, despensas y abarrotes, existen aproximadamente 20 establecimientos distribuidos en la localidad y de estos 2 llegan a ser los más destacados dentro del mercado de la cabecera cantonal de Muisne.

Existen aproximadamente 10 negocios, de las llamadas tiendas de barrios, que están ubicadas en varios sectores de la localidad, estos adquieren y ofrecen principalmente productos alimenticios tales como procesados, verduras y granos; los precios de los productos son altos debido a que la adquisición de la mercadería pasa por varios intermediarios, muchas veces sus proveedores son las tiendas de abarrotes y despensas ubicadas en el centro de Muisne, mantienen muy poco stock de los productos con el consecuente impacto sobre los precios. 
Factibilidad de creación de un supermercado en el cantón Muisne de la provincia de Esmeraldas

Cuadro 1. Tiendas de Barrios

\begin{tabular}{|l|c|}
\hline \multicolumn{2}{|c|}{ No $^{\text {Negocios }}$ Tiendas de Barrio } \\
\hline Ramírez & Ubicación \\
\hline Los Pérez & Isla de Muisne \\
\hline Sra. Virginia & Isla de Muisne \\
\hline Don Lulo & Isla de Muisne \\
\hline Rojas & Isla de Muisne \\
\hline Colorado & Isla de Muisne \\
\hline América & Isla de Muisne \\
\hline Dulces de Azúcar & Isla de Muisne \\
\hline Carlitos & Isla de Muisne \\
\hline Elci & Isla de Muisne \\
\hline Total & Isla de Muisne \\
\hline
\end{tabular}

Fuente: elaboración propia

Hay aproximadamente 8 negocios, tipo abarrotes, que ofertan más variedad que las tiendas de barrios, están ubicados en la parte céntrica de Muisne, los precios varían dependiendo del costo de adquisición; la mercadería es obtenida en su mayoría de intermediarios que llegan a esta localidad a ofrecer de puerta en puerta las diferentes líneas de productos de consumo y los dueños de estos negocios escogen los artículos que deseen y les convengan, estos abarrotes manejan más volumen y variedad de líneas de mercadería debido a que sus ventas son más numerosas y los precios son más bajo en comparación a las tiendas de barrio, los productos que expenden son cárnicos, de limpieza, bebidas y legumbres, frutas, procesados, lácteos, embutidos e higiene personal.

Cuadro 2. Tiendas de Abarrotes

\begin{tabular}{|l|l|}
\hline \multicolumn{2}{|c|}{$\mathbf{N}^{0}$ de Tiendas de Abarrotes } \\
\hline Negocios & \multicolumn{1}{c|}{ Ubicación } \\
\hline Ramona Wigton & Nuevo Muisne \\
\hline Lilian Moreno & Nuevo Muisne \\
\hline Paisanos & Nuevo Muisne \\
\hline Los Pérez & Isla de Muisne \\
\hline Su Vecino & Isla de Muisne \\
\hline Clavito & Isla de Muisne \\
\hline Macheche & Isla de Muisne \\
\hline Liover & Isla de Muisne \\
\hline Total & $\boldsymbol{8}$ \\
\hline
\end{tabular}

Fuente: Elaboración propia 
Factibilidad de creación de un supermercado en el cantón Muisne de la provincia de Esmeraldas

En Muisne existen dos negocios, del tipo Despensas y Bazar que realizan ventas al por mayor y menor, una de ellas comercializa productos de primera necesidad y la otra productos de bazar, papelería y plásticos, siendo las más destacadas en este lugar ya sea por sus volúmenes de ventas y movimientos constantes de mercaderías; además, sus precios tienden a ser más bajos comparados con las tiendas de barrio y abarrotes; tienen la ventaja de contar con vehículos propios que les permite ahorrar costos de transporte y proveer la mercadería, teniendo la oportunidad de conocer mercados mayoristas donde existen variedades de productos y artículos de todo tipo de consumo yuso.

Cuadro 3. Despensa y Bazar

\begin{tabular}{|l|c|c|}
\hline \multicolumn{3}{|c|}{ Despensa y Bazar } \\
\hline \multicolumn{1}{|c|}{ Negocios } & Tipo De Competencia & Ubicación \\
\hline Comercial Elvita & Fuerte Competencia & Isla de Muisne \\
\hline La Economía & Fuerte Competencia & Nuevo Muisne \\
\hline Total & $\mathbf{2}$ & \\
\hline
\end{tabular}

Fuente: Elaboración propia

En relación a la oferta proyectada, se presenta en el cuadro 4 un promedio la venta diaria, mensual, semanal y anual de las tiendas de barrios, tiendas de abarrotes y despensa y Bazar, en la misma se indica que de la misma manera en que las familias compran los comerciantes venden, pero cada uno da sus estimaciones de valores tanto de cuanto venden los negocios y cuando compran las familias.

Cuadro 4. Ventas

\begin{tabular}{|l|c|c|c|c|}
\hline \multicolumn{5}{|c|}{ VENTAS } \\
\hline Negocios & Diarias & Semanal & Mensual & Anual \\
\hline Tiendas de Barrio & $\$ 710,00$ & $\$ 4.260,00$ & $\$ 17.040,00$ & $\$ 204.480,00$ \\
\hline Tiendas de Abarrotes & $\$ 2.600,00$ & $\$ 15.600,00$ & $\$ 62.400,00$ & $\$ 748.800,00$ \\
\hline Despensa y Bazar & $\$ 2.500,00$ & $\$ 15.000,00$ & $\$ 60.000,00$ & $\$ 720.000,00$ \\
\hline TOTAL & $\mathbf{\$ 5 . 8 1 0 , 0 0}$ & $\mathbf{\$ 3 4 . 8 6 0 , 0 0}$ & $\mathbf{\$ 1 3 9 . 4 4 0 , 0 0}$ & $\mathbf{\$ 1 . 6 7 3 . 2 8 0 , 0 0}$ \\
\hline
\end{tabular}

Fuente: Elaboración propia

En relación a la demanda, el Instituto Nacional de Estadística y Censo (2013) indican que los habitantes de Esmeraldas destinan un promedio de \$227,25 mensuales para gastos de alimentación que representa el $\mathbf{3 8 \%}$, siendo el monto más considerable de los gastos que tienen los hogares de esta provincia. A fin de conocer el nivel de consumo de la población de Muisne y poder proyectar la demanda en base al consumo familiar que tienen se tomaron algunos datos, tal como se observa enel 
Dom. Cien., ISSN: 2477-8818

Vol. 4, núm. 3, julio, 2018, pp. 95-109

Factibilidad de creación de un supermercado en el cantón Muisne de la provincia de Esmeraldas

cuadro 5, que considera la INEC como se indica en la siguiente tabla que ayudará a calcular en cifras cuanto adquieren los habitantes de la localidad.

Cuadro 5. Cálculos de la demanda

\begin{tabular}{|c|c|c|c|c|c|c|}
\hline $\begin{array}{c}\mathrm{N}^{\circ} \text { de familias } \\
\text { de } 4 \\
\text { integrantes } \\
\text { promedio c/u }\end{array}$ & $\begin{array}{l}\text { \% Nivel de } \\
\text { ingresos por } \\
\text { rango }\end{array}$ & $\begin{array}{c}\mathrm{N}^{\circ} \text { de } \\
\text { familia por } \\
\text { promedio } \\
\text { de ingresos }\end{array}$ & $\begin{array}{c}\text { Promedio } \\
\text { de } \\
\text { ingresos }\end{array}$ & $\begin{array}{l}\text { Cantidad de } \\
\text { dinero } \\
\text { destinada a } \\
\text { alimentos y } \\
\text { bebidas }\end{array}$ & $\begin{array}{c}\text { Cantidad de compra } \\
\text { mensual }\end{array}$ & $\begin{array}{l}\text { Cantidad de } \\
\text { compra anual }\end{array}$ \\
\hline \multirow{4}{*}{2220} & 0,17 & 377 & $\$ 100,00$ & $\$ 38,00$ & $\$ 14.341,20$ & $\$ 172.094,40$ \\
\hline & 0,28 & 622 & $\$ 250,00$ & $\$ 95,00$ & $\$ 59.052,00$ & $\$ 708.624,00$ \\
\hline & 0,33 & 733 & $\$ 450,00$ & $\$ 171,00$ & $\$ 125.274,60$ & $\$ 1.503 .295,20$ \\
\hline & 0,23 & 511 & $\$ 550,00$ & $\$ 209,00$ & $\$ 106.715,40$ & $\$ 1.280 .584,80$ \\
\hline \multicolumn{5}{|c|}{ TOTAL } & $\$ 305.383,20$ & $\$ 3.664 .598,40$ \\
\hline
\end{tabular}

Fuente: Elaboración propia

En los resulados de la proyeccion de la demanda insatisfecha se puede notar en el cuadro 6, que los negocios existentes en muisne solo cubren el 46\% de la demanda total de la cabecera cantonal Muisne; aquí se ve la oportunidad que se aprovechará en la implantación de un Supermercado, el cual iniciará cubriendo el $25 \%$ de la demanda insatisfecha. A continuacion se presenta una proyeccion desde el 2013 al 2022 del comportamiento de la demanda que tendrá Muisne en 10 años tanto mensual como anual; además, se puede notar que hay un mercado potencial bien amplio por aprovechar, que no esta siendo completamente atendido por los ofertantes de la localidad.

Cuadro 6. Proyección de la demanda insatisfecha

\begin{tabular}{|c|c|c|c|c|}
\hline Año & \multicolumn{2}{|c|}{ Demanda Anual } & Oferta Anual & Demanda Insatisfecha \\
\hline 2013 & $\$$ & $3.664 .598,40$ & $\$ 1.673 .280,00$ & $\$ 1.991 .318,40$ \\
\hline 2014 & $\$$ & $3.752 .548,76$ & $\$ 1.713 .438,72$ & $\$ 2.039 .110,04$ \\
\hline 2015 & $\$$ & $3.842 .609,93$ & $\$ 1.754 .561,25$ & $\$ 2.088 .048,68$ \\
\hline 2016 & $\$$ & $3.934 .832,57$ & $\$ 1.796 .670,72$ & $\$ 2.138 .161,85$ \\
\hline 2017 & $\$$ & $4.029 .268,55$ & \$ 1.839.790,82 & \$ 2.189.477,74 \\
\hline 2018 & $\$$ & $4.125 .971,00$ & $\$ 1.883 .945,80$ & $\$ 2.242 .025,20$ \\
\hline 2019 & $\$$ & $4.224 .994,30$ & $\$ 1.929 .160,50$ & $\$ 2.295 .833,81$ \\
\hline 2020 & $\$$ & $4.326 .394,16$ & $\$ 1.975 .460,35$ & $\$ 2.350 .933,82$ \\
\hline 2021 & $\$$ & $4.430 .227,62$ & $\$ 2.022 .871,40$ & $\$ 2.407 .356,23$ \\
\hline 2022 & $\$$ & $4.536 .553,09$ & \$ 2.071.420,31 & $\$ 2.465 .132,78$ \\
\hline
\end{tabular}

Fuente: Elaboración propia 
Factibilidad de creación de un supermercado en el cantón Muisne de la provincia de Esmeraldas

Así mismo, producto de la consulta se pudo confirmar que las preferencias de consumo de los habitantes del cantón Muisne son productos alimenticios y artículos necesarios para el uso y consumo de sus hogares, el $60 \%$ de los habitantes realizan sus compras en Muisne son mujeres, a su vez la estabilidad económica de la población de ese cantón es media baja debido al nivel de ingresos que perciben mensualmente que les permite adquirir solo productos necesarios para el consumo de sus familias y a las actividades laborales que son negocios pequeños propios e informales, a más de éstas familias también hay grupos familiares que perciben ingresos estables que les permite también adquirir productos de bazar, ropa, accesorios entre otros.

Es por ello que se espera se logre implementar un Supermercado acorde a las necesidades y consumo de las personas que habitan en este sector, aprovechando el abastecimiento que se puede brindar al implementar un lugar de abasto que cuente con personal capacitado que dé una atención adecuada y los requerimientos necesarios que exijan los consumidores.

Por otro lado, se determinó que en la cabecera cantonal de Muisne no existe un lugar de expendio que ofrezca todo tipo de artículos de consumo en un solo lugar y sobre todo en óptimas condiciones, tales productos como: cárnicos, frutas, vegetales, de limpieza, procesados, aseo personal, vestuario, confitería, granos y secos, procesados, lácteos, embutidos, etc.; según la información receptada de las entrevistas de a los negocios indicaron, que de la población que habitan en la cabecera cantonal de Muisne, el $15 \%$ de las familias consumen productos de marcas reconocidas ya que estos tienen una medida económica media - baja y el $85 \%$ su medida es baja, es decir solo adquieren productos acorde a sus ingresos y necesidades.

Es por ello que se hace necesario implementar un Supermercado que reúna en un solo sitio una gama de artículos de consumo con infraestructura adecuada, variedad de productos acorde a las necesidades de las personas que habitan en el sector, con precios cómodos, dándole la oportunidad al cliente de recorrer por toda las instalaciones del negocio realizando un paseo de compras que les permita seleccionar, los producto que necesiten y siendo ellos mismo quienes decidan que comprar, cuanto, y a qué precio, debido a que se ofrecerá variedad de los mismos.

Se constató que el $90 \%$ de los productos que se expenderán en un supermercado tienen un periodo corto de duración que varían entre 2 a 3 días, meses y año; pero debido a la rotación que éstos tendrán 
Factibilidad de creación de un supermercado en el cantón Muisne de la provincia de Esmeraldas

al ser productos perecederos tales como alimenticios, de aseo personal, limpieza, entre otros, sus salidas serán constantes al ser productos de consumos necesarios.

\section{Conclusiones}

Dado el comportamiento de compras de las personas que habitan en Muisne y la alta aceptación por los habitantes, que se estructure un supermercado que esté acorde a sus necesidades, siendo un mercado estratégico que se espera aprovechar y explotar.

Los precios de los productos por parte de las tiendas de barrios son altos debido a que la adquisición de la mercadería pasa por varios intermediarios, muchas veces sus proveedores son las tiendas de abarrotes y despensas ubicadas en el centro de Muisne, mantienen muy poco stock de los productos con el consecuente impacto sobre los precios.

Los negocios tipo abarrotes, ofertan más variedad que las tiendas de barrios, los precios varían dependiendo del costo de adquisición; y manejan más volumen y variedad de líneas de mercadería debido a que sus ventas son más numerosas y los precios son más bajo en comparación a las tiendas de barrio.

Los negocios, del tipo Despensas y Bazar realizan ventas al por mayor y menor, sus precios tienden a ser más bajos comparados con las tiendas de barrio y abarrotes; tienen la ventaja de contar con vehículos propios que les permite ahorrar costos de transporte y proveer la mercadería.

En relación a la demanda, en la provincia la alimentación representa el monto más considerable de los gastos que tienen los hogares de esta provincia y en los resulados de su proyeccion se pudo comprobar que los negocios existentes en Muisne no cubren la demanda total de la cabecera cantonal, por lo que existe un mercado potencial bien amplio por aprovechar, que no esta siendo completamente atendido por los ofertantes de la localidad.

Se confirmo que las preferencias de consumo de los habitantes del canton Muisne son productos alimenticios y articulos necesarios para el uso y consumo de sus hogares y no existe un lugar de expendio que ofrezca todo tipo de artículos de consumo en un solo lugar y sobre todo en óptimas condiciones. 
Factibilidad de creación de un supermercado en el cantón Muisne de la provincia de Esmeraldas

\section{Referencias Bibliográficas}

INEC. Instituto Nacional de Estadística y Censo. (2014). Estadísticas. Extraído el 14 de enero del

2013. Disponible en: www.inec.gob.ec/

McCarthy, J (2005). Marketing. [en línea], Disponible en: https://www.google.com.ec/search?q=jerome+Mccarthy\&oq=chrome.69i57j015.16004j0j7\&sourcei $\mathrm{d}=$ chrome $\& \mathrm{ie}=\mathrm{UTF}-8$

Oster, M. (2013). En Ecuador hay espacio para más supermercados. [Versión electrónica]. Revista Lideres.ec. [en línea], Disponible en: http://www.revistalideres.ec/mercados/Ecuador-espaciosupermercados_0_841115888.html

Philip, K. (2005). Estrategias de marketing. México, Segunda edición.

Sánchez, S (2006). Estrategias de mercado. [Versión electrónica]. promonegocios.net. Extraído el 30 de octubre del 2012 [en línea], Disponible en: http://www.promonegocios.net

Santillán, C. A. (2012). Factibilidad para la creación de tres tiendas al paso que comercialice víveres de consumo masivo. Esmeraldas: Biblioteca PUCESE.

Urbina, G. (2010). Evaluación de Proyectos. Sexta edición. México, McGraw Hill. 